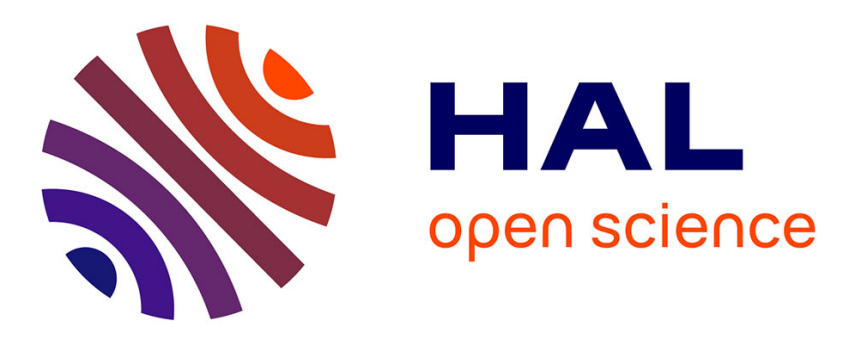

\title{
Compact and versatile OPG-OPA based on a periodically poled nonlinear crystal pumped by femtosecond Ytterbium fiber laser
}

Valerian Freysz, Gabriel Amiard-Hudebine, Yoann Zaouter, Eric Freysz

\section{- To cite this version:}

Valerian Freysz, Gabriel Amiard-Hudebine, Yoann Zaouter, Eric Freysz. Compact and versatile OPGOPA based on a periodically poled nonlinear crystal pumped by femtosecond Ytterbium fiber laser. Photonic West 2019, SPIE, Feb 2019, San Francisco, United States. 10.1117/12.2505769 . hal02358935

\section{HAL Id: hal-02358935 \\ https://hal.science/hal-02358935}

Submitted on 12 Nov 2019

HAL is a multi-disciplinary open access archive for the deposit and dissemination of scientific research documents, whether they are published or not. The documents may come from teaching and research institutions in France or abroad, or from public or private research centers.
L'archive ouverte pluridisciplinaire HAL, est destinée au dépôt et à la diffusion de documents scientifiques de niveau recherche, publiés ou non, émanant des établissements d'enseignement et de recherche français ou étrangers, des laboratoires publics ou privés. 


\title{
Compact and versatile OPG-OPA based on a periodically poled nonlinear crystal pumped by femtosecond Ytterbium fiber laser
}

\author{
Valerian Freysz ${ }^{\mathrm{a}}$, Gabriel Amiard-Hudebine ${ }^{\mathrm{a}}$, Yoann Zaouter ${ }^{\mathrm{b}}$, and Eric Freysz ${ }^{\mathrm{a}}$ \\ ${ }^{a}$ Univ. Bordeaux, CNRS, LOMA UMR 5798, 33405 Talence (France) \\ bAmplitude Systèmes, Cite de la Photonique, 33600 Pessac (France)
}

\begin{abstract}
A $10 \mathrm{~mm}$ long PPLN crystal pumped by $125 \mathrm{~nJ}, 250$ fs pulses centered at $1035 \mathrm{~nm}$ yielded by Yb3+ femtosecond fiber oscillator generates femtosecond signal and idler pulses tunable in the $1.35 \mu \mathrm{m}-1.65 \mu \mathrm{m}$ and $2.6 \mu \mathrm{m}-$ $4.2 \mu \mathrm{m}$ spectral ranges. A numerical model accounting for both second- and third-order nonlinear processes well agree with the recorded signal conversion efficiency (up to $42 \%$ ), the spectral and temporal profile of the generated pulses. Pulse to pulse stability is drastically improved injecting this compact and versatile device with a continuum generated in a photonic fiber. Further improvements are discussed.
\end{abstract}

Keywords: Optical parametric amplification, Parametric generation, Three-waves mixing, Femtoseconde pulse, Chirped pulse, Ytterbium fiber laser, PPLN crystal, Continuum generation

\section{INTRODUCTION}

A large variety of applications requires stable, coherent, high repetition rates and widely tunable femtosecond light sources with high peak intensities. The progress of high average power passively mode locked solid state and fiber laser have triggered the development of optical parametric oscillators (OPOs), optical parametric generators (OPGs) and optical parametric amplifiers (OPAs). The latter devices make it possible to down convert the frequencies of laser in the IR spectral range. Compared to OPO the design of an OPAs is simpler. OPA requires the use of a seed source which can tune continuous laser, an OPG or a white light continuum produced by the pump pulses. Double pass OPG-OPA configuration has been shown to provide compact, simple and versatile system. The main drawback of this system is the pulse to pulse fluctuations which can be strongly reduced by the system close its saturation. Along this line, we present hereafter the OPG-OPA we developed using a 10 $\mathrm{mm}$ long PPLN crystal which is pumped by an ytterbium fiber laser. The characteristics, performances of this device are discussed. A numerical model accounting for both second- and third-order optical nonlinear processes account very well for our experimental data. Further improvements of this device are discussed.

\section{EXPERIMENTAL SET UP AND RESULTS}

\subsection{Experimental set-up.}

The OPG-OPA set-up we have developed is depicted in Figure 1. It is pumped by a laser GOJI provided by AMPLITUDE. The latter delivers at a repetition rate of $40 \mathrm{MHz}$ up to $125 \mathrm{~nJ}, 250 \mathrm{fs}$ pulses centered at $1035 \mathrm{~nm}$. A half-wave plate (1/2) and a Faraday isolator (I.O) are used to attenuate the pump beam and avoid its retro-reflection. The second half wave plate makes it possible to finely adjust the polarization of the pump pulses which is reflected by a dichroic mirror (M.D) and focused by $\mathrm{f}=200 \mathrm{~mm}$ lens (F1) into the $10 \mathrm{~mm}$ long $\mathrm{MgO}$ doped periodically poled lithium niobate (PPLN) crystal. The focal lens was chosen to optimize the optical

Further author information: (Send correspondence to Eric Freysz.)

Valerian Freysz.: E-mail: valerian.freysz@u-bordeaux.fr

Gabriel Amiard-Hudebine.: E-mail: gabriel.amiard-hudebine@u-bordeaux.fr

Yoann Zaouter.: E-mail: yoann.zaouter@amplitude-laser.com

Eric Freysz.: E-mail: eric.freysz@u-bordeaux.fr, Telephone: + 33 (0)5 40008313 
parametric generation within the PPLN while keeping the peak pump power density below its damage threshold. The PPLN was provided by Covesion Ltd. The $1 \mathrm{~mm}$ thick PPLN has 9 different poling channels (poling period $\Lambda=27.91,28.28,28.67,29.08,29.52,29.98,30.49,31.02$ and $31.59 \mu \mathrm{m})$ for quasi-phase matching along the z-axis. The end facets are antireflection coated $(\mathrm{R}<1.5 \%$ @ $1064 \mathrm{~nm}, \mathrm{R}<1 \%$ @1400-1800 nm and R 6\%-3\% @ $2600-4800 \mathrm{~nm}$ ). The crystal is mounted into an oven which temperature is controllable from $30^{\circ} \mathrm{C}$ to $250^{\circ} \mathrm{C}$. A micrometric translation stage adjusts the position of the different poling gratings with respect to the pump beam. After the crystal the pump and signal waves are separated by a second dichroic mirror. The pump and signal beams are then collimated, reflected and refocused into the PPLN crystal (F2 for the pump and F3 for the signal). The delay between the signal and pump pulses is adjusted by moving one of the reflecting mirrors. The signal and idler pulses generated during the second pass in the PPLN crystal are transmitted by the first dichroic mirror.

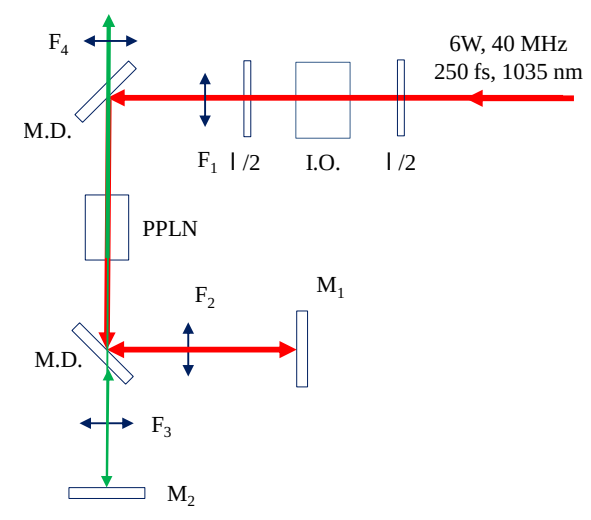

Figure 1: Experimental setup. Half-wave plate (1/2). Faraday isolator (I.O). Dichroic mirror (M.D). Lens (F)

\subsection{Experimental results.}

The conversion rate of the pump to the signal in the PPLN is presented in Figure 2a. From 1.3 $\mu \mathrm{m}$ to $1.48 \mu \mathrm{m}$, conversion is around $40 \%$. Then, it decreases gradually as the wavelength increases and reaches $10 \%$ at $1.6 \mu \mathrm{m}$. The average power of the signal pulses centered at $1.4 \mu \mathrm{m}$ pumped with $4 \mathrm{~W}$ is then about $1.6 \mathrm{~W}$. The spatial profile of the beam is very close to the $\mathrm{TEM}_{00}$ mode of the pump beam (Figure 3). We also measured the spectrum and autocorrelation trace of the signal pulses generated over the entire spectral range covered. The duration at full width at half maximum (FWHM) (assuming Gaussian shape) of the signal pulses varies between $450 \mathrm{fs}$ and $205 \mathrm{fs}$. The FWHM of the spectrum (Figure $2 \mathrm{~b})$ goes from $5 \mathrm{~nm}$ for $27.91 \mu \mathrm{m}(\sim 1400 \mathrm{~nm})$ poling period to $27 \mathrm{~nm}(\sim 1625 \mathrm{~nm})$ for $30.49 \mu \mathrm{m}$.

\subsubsection{Injection of OPA by a super continuum generated in a fiber.}

As shown by Linnenbank and Linden, ${ }^{1}$ the lower the conversion efficiency of the OPA, the greater the pulseto-pulse intensity fluctuations of the signal pulses. This phenomenon is simply explained. It is related to the parametric fluorescence generation of the signal wave during the first passage of the pump pulse in the crystal. This phenomenon is, by nature, extremely noisy. To limit these fluctuations, the amplification of the pulse signal during its second passage in the crystal must be made in the vicinity of the parametric gain saturation threshold : where the conversion efficiency is optimal.

To limit these fluctuations, one solution is continous wave (CW) injection seeding. ${ }^{2}$ Another solution is to use a supercontinuum. ${ }^{3}$ We have tested the last solution and injected the OPA with a super continuum generated in a sufficiently long fiber $(\sim 1 \mathrm{~m})$. In this case, even if the conversion efficiency is low, the amplitude noise of the injected signal pulses is greatly reduced and the amplitude noise of the amplified pulses by the OPA is much smaller. The parametric amplification of a signal pulse produced by parametric generation or a super continuum is presented on the Figure 4. It is noted that the implementation of the super continuum greatly limits fluctuations. It should be noted that to carry out this measurement we used a fiber whose dispersion was not negligible at the wavelength of the pump. 


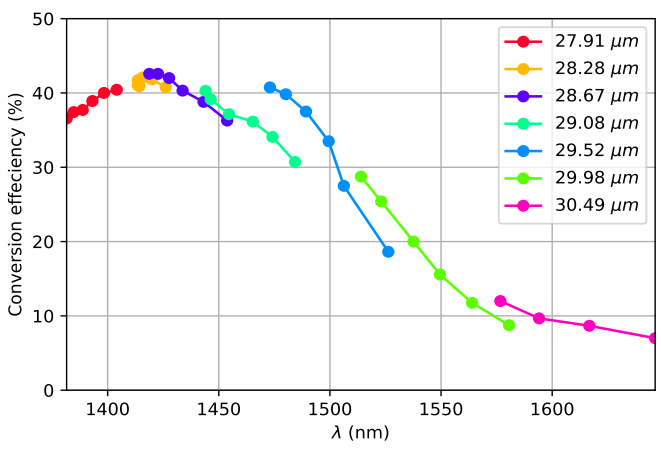

(a) Signal conversion efficiency versus wavelength for different grating periods.

Figure 2: Signal conversion and spectrum for different grating periods.

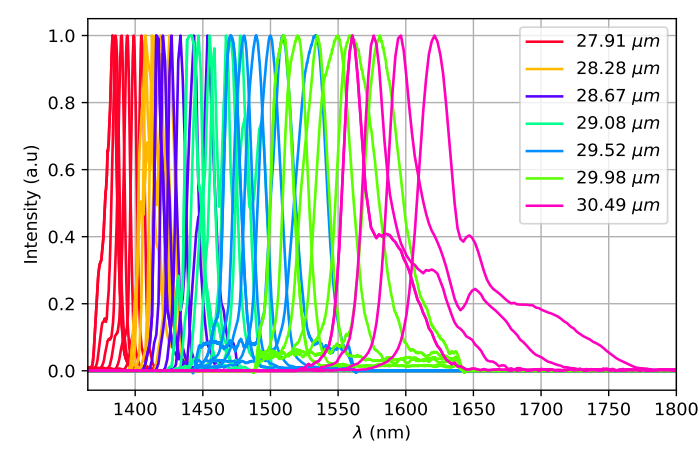

(b) Spectrum for different grating periods.

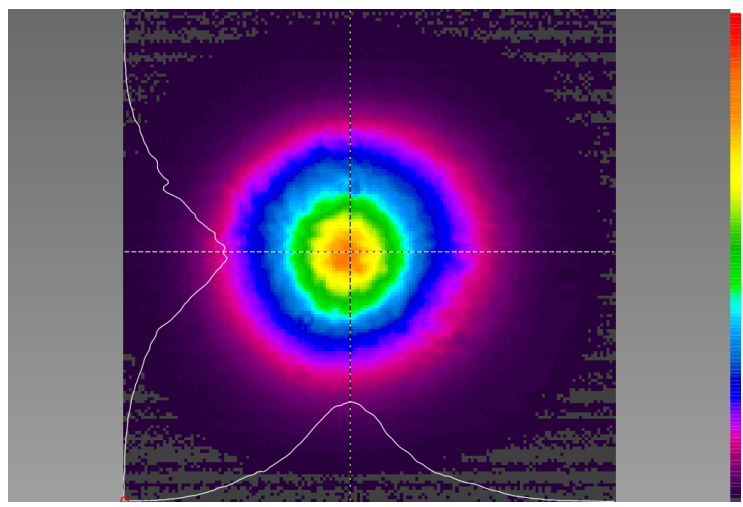

Figure 3: Typical spatial profile for the signal after two pass in the PPLN.

\subsection{Numerical simulations and comparison with the experimental data.}

We modeled the pulse propagation in the PPLN crystal. Our model uses pyNLO python library "Nonlinear optics modeling for Python". The solver used makes it possible to account for three waves and third order non-linear effects. The current version of this solver (0.1.2) contains an error on the coefficients of Sellmeier of the lithium niobate which we corrected. ${ }^{4}$ In particular, our simulations carried out at a temperature of $210^{\circ} \mathrm{C}$, calculate the conversion efficiency, the spectrum and the autocorrelations trace of the signal and complementary pulses. Hereafter, we illustrate the ability of this numerical model to report our experimental results considering two cases, trapping and self-compression.

\subsubsection{The trapping regime.}

Xie and al. ${ }^{5}$ showed numerically that it was possible to obtain a signal with pulses close to the Fourier transform limit when $\left|\delta n_{s p} / \delta n_{s i}\right|<1$ with $\delta n_{s p}=\left|n_{s}^{g}-n_{p}^{g}\right|$ and $\delta n_{s i}=\left|n_{s}^{g}-n_{i}^{g}\right|$ where $n_{j}^{g}$ is the group index and s,i,p represents the signal, idler and pump respectively. In this trapping regime the pump pulse is framed by the signal pulse and the idler pulse. This increases the temporal overlap of the pump with the signal ans idler pulses. It makes possible to generate quasi-Fourier transformed pulses while optimizing the conversion efficiency.

The calculated pump-signal conversion efficiency is $\sim 60 \%$ which is slightly greater than the measured conversion efficiency of $\sim 40 \%$. It can be seen that the spectrum and the autocorrelation trace simulated and measured have particular and similar forms that can be explained by the simulation. The figure 6 shows the pump, signal and complementary pulses as well as the signal spectrum at two different times in the crystal. As we can see by comparing figure $6 \mathrm{~b}$ and figure $6 \mathrm{a}$, the slight staircase on the figure 5 is typical of a reconversion of the 


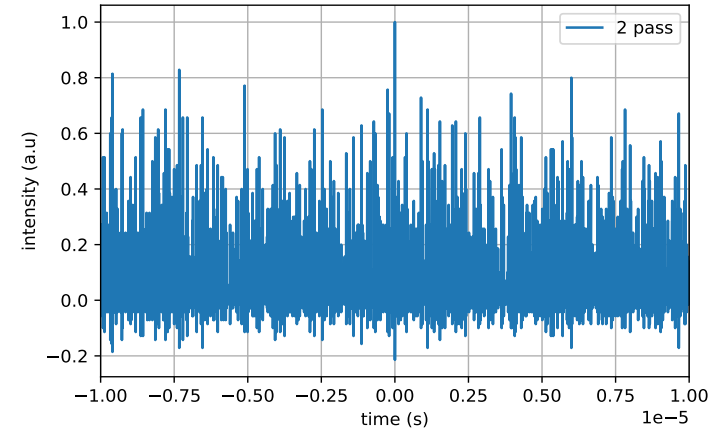

(a) Measurement of a Signal Pulse Train Produced by Parametric Fluorescence and Amplified by OPA Outside Saturation.

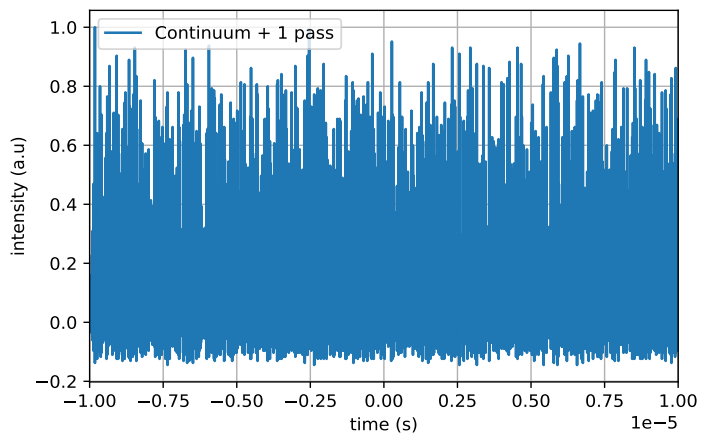

(c) Measurement of a pulse train produced by a supercontinuum and amplified by the OPA outside saturation.

Figure 4: Comparison of a weakly amplified signal pulse train produced by parametric generation and a supercontinuum.

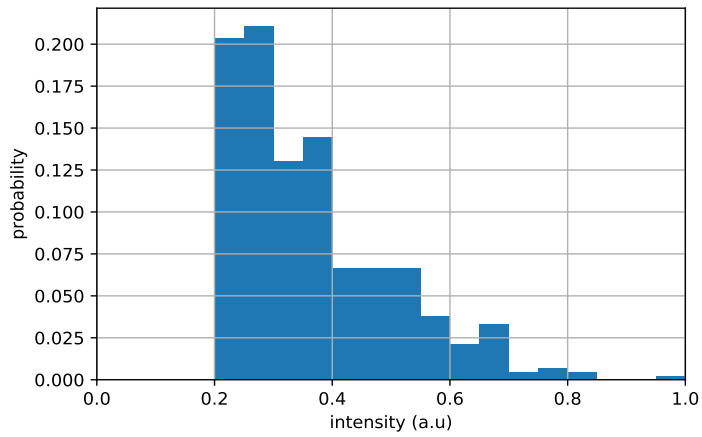

(b) Statistic of the signal pulse train produced by parametric fluorescence and amplified by the OPA outside saturation.

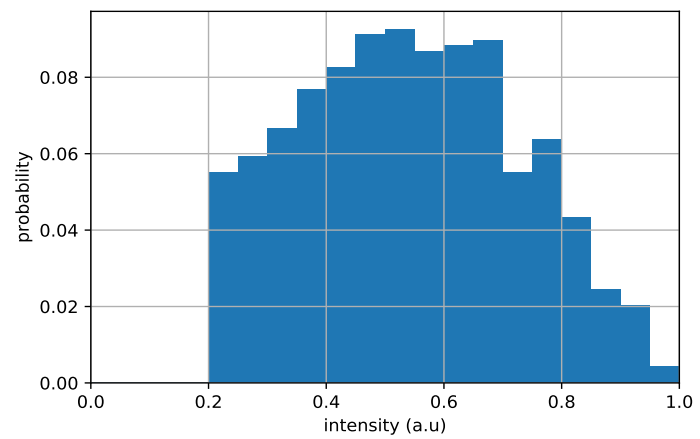

(d) Statistic of a pulse train produced by a supercontinuum and amplified by OPA out of saturation. 
pump. Specifically, this indicates that to have the maximum conversion we should slightly reduce the overlap time between pump and signal pulses. This example stress how our modeling makes it possible to optimize OPA or OPO.

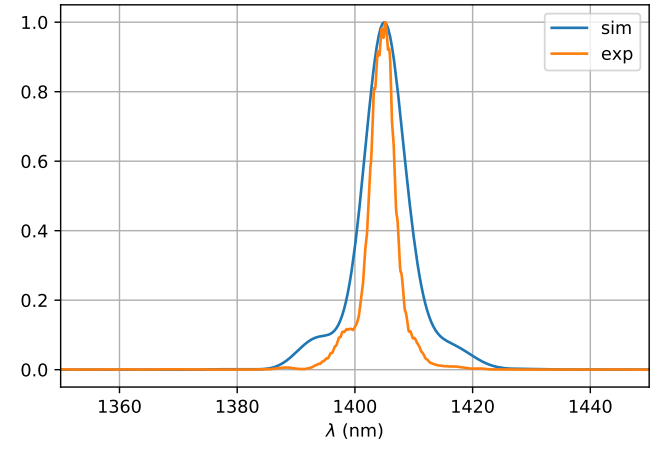

(a) Spectrum

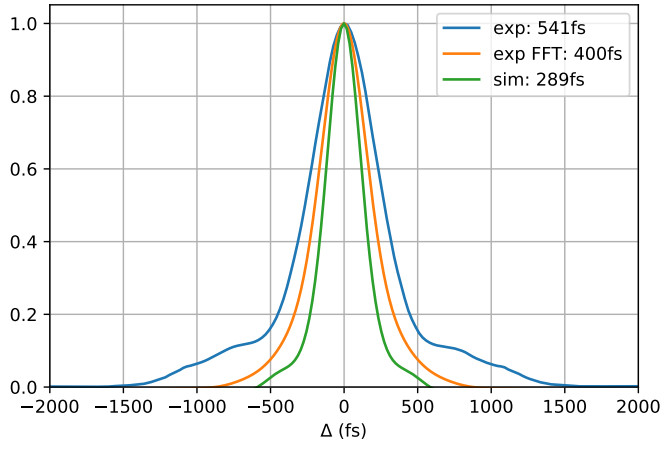

(b) Autocorrelation

Figure 5: Comparison of simulation and experimental measurement for the grating periods $27.91 \mu \mathrm{m}$ à $180^{\circ} \mathrm{C}$ with a average pump power pump $3.5 \mathrm{~W}$.
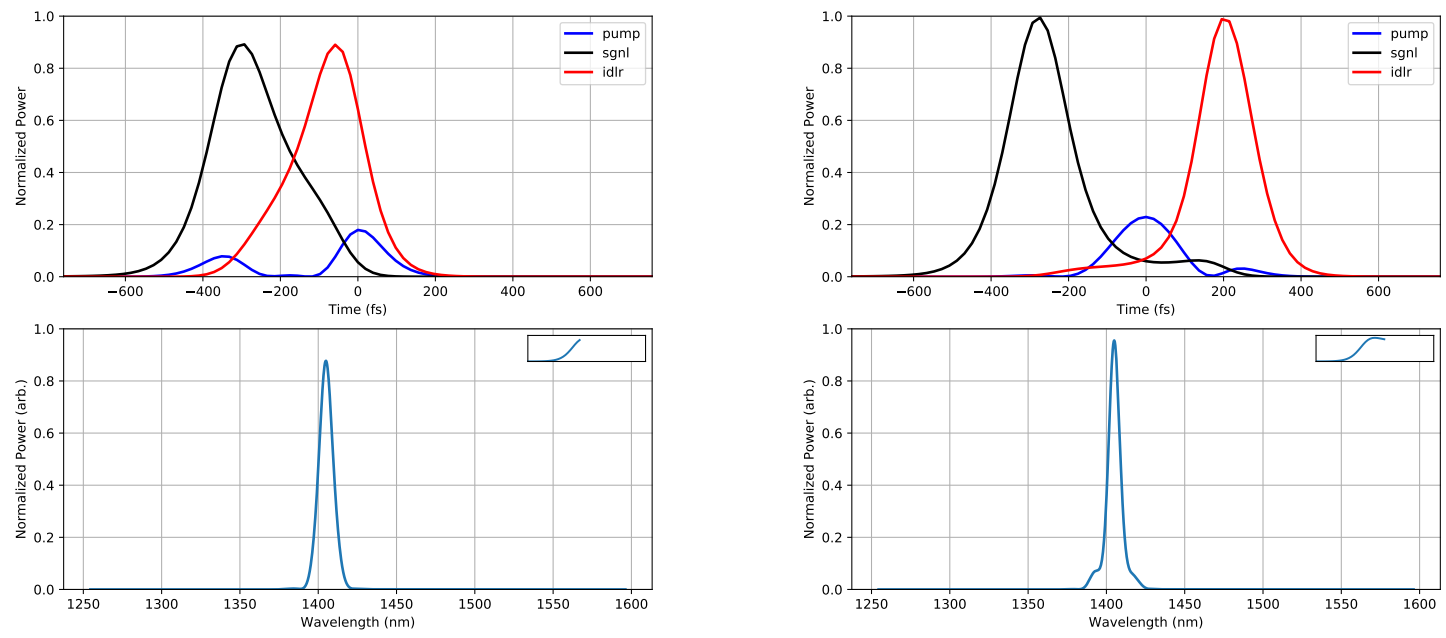

(a) $14.68 \mathrm{ps}$

(b) $19.35 \mathrm{ps}$

Figure 6: Spectrum of the signal and pulse pump, signal and idler in the crystal at two different times for the grating periods $27.91 \mu \mathrm{m}$ at $180^{\circ} \mathrm{C}$ with an average pump power pump $3.5 \mathrm{~W}$.

\subsubsection{The self-compression regime.}

Here, we demonstrate that it is possible to generate a signal pulse having a shorter duration than that of the pump pulse. This result is illustrated with the grating $29.52 \mu \mathrm{m}$ at $180^{\circ} \mathrm{C}$.

The spectrum presented on figure 7a have a FWHM of $23 \mathrm{~nm}$. The autocorrelation trace of the signal pulses obtained with this grating is presented on the Figure $7 \mathrm{~b}$. The FHWM duration for the signal pulse is $287 \mathrm{fs}$ while that for the pump pulse is $358 \mathrm{fs}$. It should be noted, however, that in this regime the pump-signal efficiency is lower than in the trapped one, with a pump to signal conversion efficiency around $20 \%$. 
The calculated pump-signal conversion efficiency is $19.6 \%$. This is in good agreement with the measured efficiency of $20.9 \%$. The comparison of the crystal output spectra and the autocorrelation traces is presented on the Figure 7. It emphasizes here again the good agreement between experience and simulation.

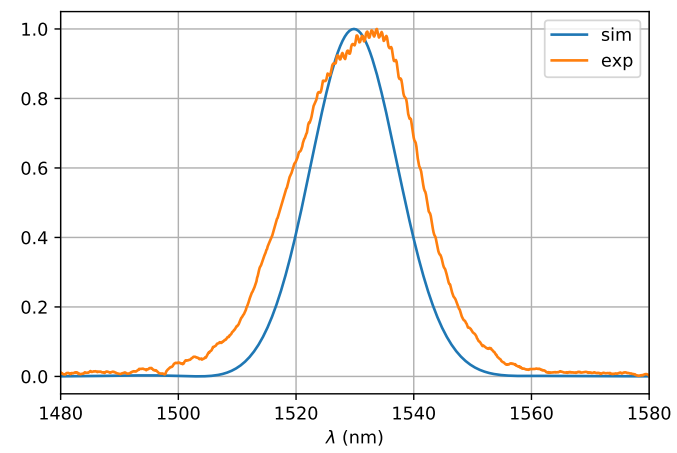

(a) Spectrum

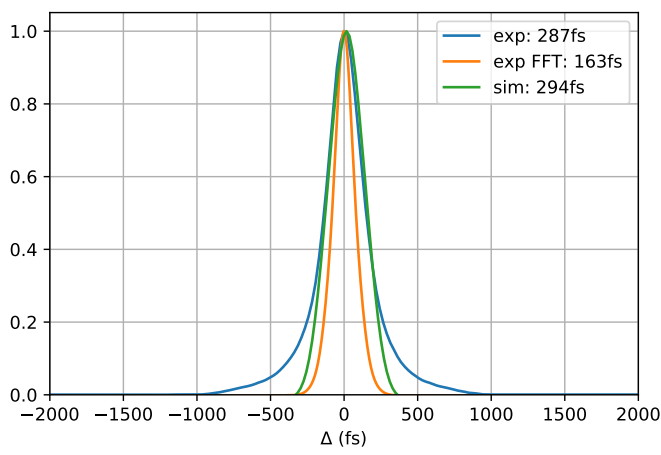

(b) Autocorrelation

Figure 7: Comparison of simulation and experimental measurement for the grating periods $29.52 \mu \mathrm{m}$ at $180^{\circ} \mathrm{C}$ with a average pump power pump $4 \mathrm{~W}$.

\section{CONCLUSION}

In this paper, we have presented a near-infrared OPA pumped by a femtosecond ytterbium laser oscillator delivering at $40 \mathrm{MHz}$ pulses with a duration of $\sim 250 \mathrm{fs}$ centered at $1035 \mathrm{~nm}$ having an energy of $125 \mathrm{~nJ}$. Pump-to-signal conversion efficiency are greater than $40 \%$ over spectral range from $1380 \mathrm{~nm}-1480 \mathrm{~nm}$. At higher wavelength, due to the decreasing of the interaction length, the conversion is lower. Over this wavelength conversion efficiency then decreases as the signal pump interaction length decreases. Conversion efficiency is higher than $15 \%$ up to $1600 \mathrm{~nm}$. Below this wavelength, the OPA is unstable. This is likely due to the strong second harmonic generation of the pump that induces photo-refractive effects in the PPLN crystal. The beam quality of the signal pulse is very good and is directly related to the pump beam quality.

Many improvements can be made. First of all, the perfect and systematic manual synchronization between signal and pump pulses is hard to achieve and can lead to unreliable results. To overcome this constraint, we have built an auto-tune synchronization based on a lock-in amplifier and a nano-scale precision linear stage. We will also test our OPA using a Lithium tantalate crystal $\left(\mathrm{LiTaO}_{3}\right)$, in fan out configuration. It will select the signal wavelength via a simple translation and will significantly reduce the time response. Our calculations indicate that the temporal trapping regime of the signal pulse should be observed over a broad wavelength range. This will result in significant conversion efficiency over a wider spectral range. Finally, we plan to use an NKT SC-5.0-1040-PM fiber with zero dispersion at $1040 \mathrm{~nm}$. It will reduce the pulse-to-pulse fluctuations of the signal and complementary pulses while minimizing the fiber length required for efficient generation of super continuum.

\section{REFERENCES}

[1] Linnenbank, H. and Linden, S., "High repetition rate femtosecond double pass optical parametric generator with more than $2 \mathrm{~W}$ tunable output in the NIR," Optics Express 22, 18072-18077 (July 2014).

[2] Linnenbank, H., Steinle, T., and Giessen, H., "Narrowband cw injection seeded high power femtosecond double-pass optical parametric generator at $43 \mathrm{MHz}$ : Gain and noise dynamics," Optics Express 24, 19558 (Aug. 2016).

[3] Krauth, J., Steinmann, A., Hegenbarth, R., Conforti, M., and Giessen, H., "Broadly tunable femtosecond near- and mid-IR source by direct pumping of an OPA with a $417 \mathrm{MHz} \mathrm{Yb}: \mathrm{KGW}$ oscillator," Optics Express 21, 11516 (May 2013). 
[4] Gayer, O., Sacks, Z., Galun, E., and Arie, A., "Temperature and wavelength dependent refractive index equations for MgO-doped congruent and stoichiometric LiNbO3," Applied Physics B 91, 343-348 (May 2008).

[5] Xie, X., Schober, A. M., Langrock, C., Roussev, R. V., Kurz, J. R., and Fejer, M. M., "Picojoule threshold, picosecond optical parametric generation in reverse proton-exchanged lithium niobate waveguides," Journal of the Optical Society of America B 21, 1397 (July 2004). 